\title{
Leprosy at the interface between epidemiology and basic research
}

\author{
MF LECHAT
}

Ecole de Santé Publique, Epidémiologie - UCL 30.34, Clos Chapelle aux Champs 30, 1200 Bruxelles, Belgique

Epidemiology has been defined as the study of diseases in populations. It is, at the same time, a tool for counting events and a window for looking at diseases. I think these two factors have been superbly highlighted during this symposium.

As a measuring instrument, epidemiology serves several purposes. In defining the magnitude of the problems it helps in planning and setting priorities. Trends of frequencies can be predicted. It provides baselines for evaluation of control strategies.

With respect to the magnitude of the leprosy problem according to the most recent statistics from WHO, there are an estimated 10-11 million patients in the world, some 5 million of them registered, half of those in India. And although those figures cannot be compared to other communicable diseases such as malaria and schistosomiasis, what makes leprosy work a priority is the high frequency of severe disabilities in the patients. There are also large geographical differences, even on the small scale, resulting in a patchy distribution of prevalences for which no epidemiological explanation is readily available. The total number of patients in the world during the last two decades or so, apparently has not changed, but this is partly due to the combined effects of better case-finding, lower mortality, differences in diagnostic criteria, confusion of epidemiological rates, and population increase. In a few countries where effective control measures have been properly enforced, prevalence and/or incidence have actually decreased, at times in considerable proportions.

For many years one major deficiency of leprosy control has been the lack of an appropriate information system, including a standardized methodology to properly collect, record and retrieve epidemiological and operational leprosy statistics. Also a standard definition of clinical leprosy should be agreed upon, especially in its very early forms. This has not prevented the collection of excellent data in a number of countries, e.g. in India and in Japan, among others. There is, however, a need to define a minimum set of basic data required to monitor leprosy control and provide baselines for ad hoc studies and trials, which could be used under field conditions. One could much lament about the undoubtedly junk quality of 
most of the epidemiological data presently available, but I think a constructive effort should be attempted to outline the type of information actually needed. Much was said about the confusion between incidence and case-detection rates, although it is now possible with the use of appropriate indices to control and reduce this confusion. As shown in several presentations, age, sex, or type specific incidence/case-detection rates provide useful hints as to the dynamics of the disease, but as shown in Norway, linkage of these factors can easily be made to provide age-sex-type specific indices, which considerably increase their value to predict future trends.

Let us now turn to the second facet of epidemiology - epidemiology as a window. There is no window without a frame, except in surrealistic paintings, and there is no epidemiology in a vacuum. Figures and indices, rates and ratios, as well defined as they can be, come to life only when bred with and built on basic research. Epidemiological determinants are classically divided into three groups: agent factors, host factors, and environmental factors.

It is, perhaps, some kind of an illusion to chase environmental risk factors in leprosy when there are so many confounding variables and we have so few hard facts to feed on. There is only one snow on cholera. Looking for environmental determinants in leprosy makes me think at times of the chap who discovering that both merchant seamen and playboys on their yachts have syphilis, would have concluded that seawater is the agent.

In fact, the contribution of epidemiology to our knowledge of leprosy has been remarkably meager. The study by coull and Ginto of attack rates in household contacts according to the type of the indexcase - one of the classics of epidemiology - has been repeatedly mentioned, but that is about it.

Microbiology, of course, has provided one of the missing legs. On February 28, 1873 - so we learned - Hansen for the first time saw the bacillus. Past and present knowledge of the microbiology of $\mathrm{M}$. leprae has been assembled, and a clearer picture is emerging. We have good evidence regarding the source of infection, the ulcerated nose of the bacilli-discharging patients, and possibly other secretions. The rôle of carriers as the source of infection is not elucidated, in spite of its potential importance for transmission. Observations, made by Chatterjee, and Figueredo and Desai in India years ago, of bacilli in or on the skin of a significant proportion of contacts should be repeated using the present criteria available

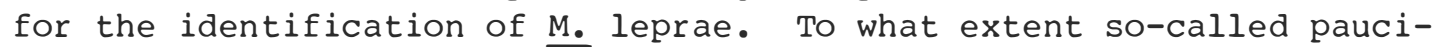
bacillary leprosy may serve as a source of infection is still an unresolved question. We are far from sure about the portal of entry, broken skin, respiratory tract, or both. We have discovered that M. leprae may survive in moist dust for over six weeks, and this opens broad perspectives as regards transmission through indirect contact, degree and type of exposure, transport, dissemination, hapitat, hygiene, and the like. We know close to nothing regarding the doses needed for infection to take place, and more data should be collected along this line, possibly on the model of what has been 
done for tuberculosis. The possibility of an occasional or unsuspected usual animal reservoir still deserves investigation.

But then comes the gap. Here is the bacillus in the wild looking for a man, and there is the patient, one, two, ten, or twenty years later, with the whole range of the Ridley-Jopling spectrum to choose from.

In the meantime, nobody knows. One cannot even talk about this interval because until recently there was no way to recognize infection from non-infection. This is probably what has made epidemiology so relatively inefficient. The end point of any epidemiological study in leprosy was by necessity the overt disease, and how are we to relate this to events that have occurred several years earlier and cannot even be identified in time?

The development of immunological tests to recognize infection would therefore be a major break-through. The lymphocyte transformation test (LTT) of Godal and Negassi for cell mediated immunity or reactivity and the serological tests developed in Norway (radioimmunoassay) and in Japan (immunofluorescence) are steps in the right direction.

To serve a useful purpose, however, these tests have to be not only highly specific, but techniques also have to be developed to make them practicable under field conditions.

With these tests, provided they have an acceptable specificity, the epidemiology of leprosy-disease may now turn into an epidemiology of leprosy-infection. At the moment, applied on a population basis, they could tell us how many are infected, when they were infected, and under what circumstances, e.g. whether people migrating to a leprosy environment soon become infected, whether most of the people in endemic areas are infected compared with a very few who eventually develop the disease, whether leprosy is an occupational risk, whether infection occurs at a young age, and whether contacts are infected sooner. All this is a lot. At the moment, however, there is a crucial issue. To what extent they are predictors of the further course of events - subclinical infection, protection, selfhealing, type of leprosy, or anything - or, in other words, how is immunological activity related to prognosis after infection and to what extent do current tests provide information on the degree of protective immunity in individuals and at population levels - is apparently another matter.

I must confess that this meeting has failed to convince me that a lymphoblastic transformation is more than a marker of infection; the LTT shows that something has gone amok, that someway $\underline{M}_{\bullet}$ leprae caused it, but as to its value of indicating protection or predicting anything for the further course of events, it has apparently none. We do not even know its meaning yet regarding $\mathrm{T}$ cells and $\mathrm{B}$ cells, suppressors, killers and helpers, acting together in a kind of elegant ballet. Humoral tests do not seem to fare better. It is still much research to be done for ascertaining their predictive value. There is, therefore, no way to ascertain the relation between current tests and protective immunity at the moment, except by longitudinal studies. This is disturbing. Longitudinal studies 
of this sort will have to be extended for 10 or 20 years, which is clearly unfeasable, especially in endemic countries where follow-up studies at times raise insuperable difficulties. In the meantime vaccine field trials may be well underway. There are, however, some data of predictive value that we have seen this afternoon from the RIA which have been obtained recently in Ethiopia by repeated examinations of patients with high titres of antibodies.

The availability of markers of infection will have two major applications. First, it will become possible to apply the classical epidemiological case-control approach to investigate risk factors in the environment, e.g. the possible rôle of ecological determinants, sphagnum among others. Secondly, it should be possible to ascertain on an individual basis who is infected and who is not. This is a major point in relation to the vaccine trials now under consideration.

At the moment, the design of protocols for leprosy vaccine trials is faced with the major difficulty of having to cope with population groups whose degree of exposure and prevalence of infection cannot be ascertained, with the result that the interpretation of the possible outcomes is difficult.

The consequences of having appropriate tests to recognize leprosy infection will be incalculable. I also wish that some ancient epidemiological studies on lepromin reactivity would be repeated using these tests.

The question was raised as to what extent the specificity of the current test is meeting the requirement for epidemiological studies. It remained unanswered. In this respect, the development of monoclonal antibodies specific for M. leprae is, of course, a major priority since it will enable us to identify possible patients unequivocally rather than in terms of false positives and false negatives.

One major difficulty remains, the possibility of genetic factors intervening before infection to prevent a cellular or humoral response when the individual is exposed to M. leprae. That it could be so is suggested by the now famous cohort study of Dharmendra and Chatterjee in India, showing that leprosy occurred only in individuals who were lepromin negative. It might be that a study of the correlations of LTT and humoral response in a number of patients could help to identify peculiar groups of individuals, LTT negative and with high antibody titres, in which it would be worthwhile to look for genetic patterns, HLA or non-HLA antigens, or cellular radiosensitivity related to DNA processing.

Now, why infected people with subclinical leprosy or in the incubation-latency stage (whatever we choose to call it) eventually develop the disease is quite another matter. This is the epidemiology of leprosy-disease in infected people, and at this stage I think we better leave it to the immunologists, in the first place, and to microbiologists and pathologists. It might be that the fate is decided at the time of infection.

Non-specific factors such as bouts of secondary diseases, malnutrition, lactation and pregnancy have been mentioned as possible determinants of clinical disease; Such factors can be studied by 
classical epidemiological methods, both ny case-control and longitudinal studies. The possibility of a lymphocytotrophic virus infecting clones of activated $T$ cells at the right moment to blank the whole system has been mentioned. No doubt, there is a long way to go - and a fascinating one - to explain why subclinical infection turns into clinical disease in some unfortunate individuals. Here again the availability of monoclonal antibodies may revolutionize the whole approach, making it possible to follow the fate of $M$. leprae in host. By the same token, it will resolve the current controversy about latency versus incubation period.

The major contribution, however, that we epidemiologists can bring to the epidemiology of leprosy-disease, and an essential contribution, is the collection of good population data to formulate and test hypotheses. This can be conducted in two directions.

One would be to provide a minimum set of valid, reliable, and manageable epidemiological data, e.g. attack rates during pregnancy and lactation, incidence of secondary diseases, and measurements of environmental factors - all those factors which would possibly trigger the shift from subclinical infection to clinical disease. There is, indeed, as already said, a great need for basic epidemiological data, collected on a standardized basis, in leprosy-prevalent areas. Another contribution we could make, and I think time is running short for this, is to compile an inventory of special communities with peculiar characteristics for leprosy. It is a pity that we did not have the tools we have today to investigate the Nauru epidemics. But there are a number of such communities in the world; some were mentioned, especially in the Americas and in India, but there are others, and they are fast disappearing. These communities could constitute areas of selection for joint research by epidemiologists, immunologists, and geneticists. Such studies could help us to document present hypotheses regarding the various suspected patterns of the dynamics of leprosy under different conditions, epidemics or endemics, rural or urban, and why it is not developing in some areas or under some conditions (West Coast of Africa, reintroduction by immigrants) and why it is disappearing in others. What occurred in Norway - and has been so wonderfully and extensively studied - is occurring now in a number of places in the world. This is a wide field for research that could be useful in the future for handling of leprosy in those unfortunate countries where, at times, in spite of frantic efforts to control leprosy, prevalence still shows no or little changes.

There is a great need, indeed, for joint immuno-epidemiological studies in leprosy. Some time we should meet to make a list of relevant and feasible studies of that sort and establish some kind of coordinated strategy for these efforts.

Now, I should turn to another issue of major importance for leprosy control, that is the emergence of a new disease with peculiar characteristics, namely drug-resistant leprosy. The discovery of mice as a suitable animal for inoculation has revolutionized the whole approach to leprosy, including epidemiology. It has now be- 
come possible to test the bacilli for viability, to assess drugs, and last but not least to ascertain drug resistance. Resistance has completely upset the strategy of control. While it is universally spread, estimates for its prevalence, and more especially for its incidence, still rest on rather uncertain data. It is present everywhere, but figures vary somehow, which is in fact, but in part only, due to the differences in ascertainment and sampling. What is now needed is a study of the epidemiology of dapsone-resistant leprosy, how it spread, from whom, and at what rates. For this it is necessary and urgent to establish an appropriate monitoring system with, again, standardized criteria, laboratory techniques, and recording system.

Finally, I should mention ENL and reversal reaction. These are important slits, I think, through which to look into the immunology of leprosy. Frankly, at the moment, the contribution of the epidemiologist by studying the frequency of reactions, their determinants, and identification of patients at risk has been minimal. Perhaps we should wait until clinicians have made up their minds to produce standardized definitions of these phenonemona as well as precise and unambiguous diagnostic criteria on the model of what has been done by the Ridley-Jopling classification that proved so successful for distinguishing the spectrum of the clinical aspects of the disease.

Leprosy is mostly prevalent in the poor countries of the world. It means that our methods and techniques for population-based studies should be adapted to local conditions. Sophisticated epidemiological approaches and complicated gadgetry will be of no use. Only if research develops within the constraints of these countries it could eventually benefit the some 10 million patients who are, after all, the ultimate goal and justification of research. In this respect it is most encouraging that efforts are carried out to develop procedures for the use of more sophisticated serological tests under field conditions.

It was said that our fragmentary knowledge of the epidemiology of leprosy resembles the information obtained when an elephant is touched by three blind men. It is also like a Rubik's cube with various disciplines, from microbiology to immunology, standing for the six faces. The cube is still pretty well scrambled, but, with time and experience, it will eventually be unscrambled with each colour nicely put in its place.

By taking the initiative to bring us together, the Sasakawa Memorial Health Foundation and Redd Barna - Norway have given us the opportunity to define the limits of our capacities and to turn our efforts in the right direction. On your behalf I want to express our best thanks. Than you to all of you and also to the organizors who made this meeting such a fruitful and pleasant experience. 